\title{
Major depression among methamphetamine users entering drug treatment programs
}

\author{
Rebecca McKetin, Daniel I Lubman, Nicole M Lee, Joanne E Ross and Tim N Slade
}

$\mathrm{P}$ sychotic phenomena are often considered to be the hallmark psychiatric sequelae of methamphetamine use, but depressive symptoms are far more common and can be severe and debilitating among heavy users of the drug. ${ }^{1,2}$ Although depression is not an uncommon phenomenon in drug-dependent populations, ${ }^{3}$ methamphetamine directly affects monoamine regulation within the brain, producing a pseudodepressive state that encompasses many of the features of major depression (ie, low mood, anhedonia, fatigue, sleep disturbance, appetite changes, lack of motivation, restlessness, irritability and poor concentration). ${ }^{4,5}$

If left untreated, depression can reduce adherence to drug treatment, increase the risk of relapse to stimulant use and elevate the risk of suicide. ${ }^{6,7}$ Despite this, there are no available estimates for the prevalence of major depression among methamphetamine users who enter treatment programs. Previous research has reported high levels of depressive symptoms among dependent methamphetamine users, ${ }^{7}$ and one study screened for major depression, ${ }^{6}$ but no studies have assessed the prevalence of major depression using a validated psychiatric assessment instrument. This makes it hard to determine the extent to which depression among methamphetamine users requires clinical management.

The aim of our study was to determine the prevalence of major depression over the previous 12 months in a population of dependent methamphetamine users. We used data from the baseline phase of the Australian Methamphetamine Treatment Evaluation Study, which assessed psychiatric comorbidity in a cohort of methamphetamine users entering specialised drug and alcohol services within the community.

\section{METHODS}

\section{Participants and recruitment}

Participants $(n=400)$ were recruited on entry to participating drug treatment agencies, which were selected from agencies contributing data to the Alcohol and Other Drug Treatment Services National Minimum Data Set. This includes all publicly funded government and non-government agencies

\section{ABSTRACT}

Objective: To determine the prevalence of major depression among people entering treatment for methamphetamine use.

Design, setting and participants: The study was a cross-sectional survey involving 41 specialised drug and alcohol treatment agencies in Brisbane and Sydney. Services provided by these agencies included residential rehabilitation, detoxification and counselling. Participants were 400 people entering treatment for methamphetamine use who were recruited from participating treatment agencies between January 2006 and November 2007. Participants underwent a structured, face-to-face, 1.5-hour interview. Assessment instruments included the Composite International Diagnostic Interview and the Short Form 12.

Main outcome measure: Diagnosis of a major depressive episode in the year prior to the study.

Results: The prevalence of major depression in the year prior to the study was 40\% (95\% $\mathrm{Cl}, 35 \%-44 \%)$. A noteworthy post-hoc observation was that a further $44 \%$ of participants met the symptom criteria for major depression but were excluded from a diagnosis because their symptoms were better accounted for by psychoactive substance use. Both major depression and these latter cases of "substance-induced depression" were associated with severe symptoms of depression, high levels of disability and suicidal ideation.

Conclusion: Most people entering treatment programs for methamphetamine use have levels of depression that require clinical management. Making a diagnosis of major depression in the context of heavy methamphetamine use is problematic because of substance-induced symptoms of depression.

MJA 2011; 195: S51-S55

that provide specialist treatment services to people with alcohol and other drug problems. Agencies providing counselling, residential rehabilitation and detoxification were included, but those providing assessment only, case management only or other services (eg, education) were excluded. Forty-one agencies were included in the recruitment pool - 26 in the Sydney region and 15 in the Brisbane region. These agencies included 15 outpatient counselling services, 13 residential rehabilitation facilities, 11 detoxification units and two services that provided both detoxification and residential rehabilitation.

Participants were eligible if they were entering treatment with methamphetamine or amphetamine use as a primary or secondary drug problem (78\% reported it to be their primary drug problem). Participants had to be at least 16 years of age, willing to do follow-up interviews, and able to understand English. They were excluded if, in the previous month, they had been incarcerated, had received treatment for their meth- amphetamine use, or had received any form of inpatient drug treatment. This exclusion criterion was necessary to obtain a naturalistic baseline measure of pretreatment methamphetamine use.

Participants were recruited between January 2006 and November 2007. A further 195 prospective participants were screened but found ineligible, mainly because they had been in treatment (58\%) or incarcerated (28\%) during the previous month, or had declined participation in the study (10\%).

The main treatment modalities received by participants in the final sample were residential rehabilitation $(n=211)$, withdrawal management $(n=149)$ and counselling $(n=40)$.

All participants were volunteers who provided informed consent and were reimbursed $\$ 30$ for their time and travel expenses.

\section{Procedure}

Participants took part in a structured, faceto-face, 1.5-hour interview a median of 4 
days after treatment entry (range, 0-32 days). The interview included questions on demographics (such as age, sex, employment status, country of birth, prison history, income, and living arrangement), the Composite International Diagnostic Interview (CIDI), the Short Form 12 (SF-12) and the Opiate Treatment Index (OTI). ${ }^{8}$ All responses were completed by the interviewer. Interviewers had undergone certified training in use of the CIDI.

\section{Measures}

\section{Major depression}

The CIDI was used to establish a diagnosis of a major depressive episode in the preceding 12 months, based on criteria specified in the Diagnostic and statistical manual of mental disorders, 4th edition (DSM-IV). ${ }^{9}$ The CIDI is a fully structured, lay-administered interview schedule that has been validated against clinician-administered psychiatric interview schedules and is widely used in epidemiological studies. ${ }^{10}$ In order to meet the DSM-IV criteria for a major depressive episode, five or more symptoms of depression must have been present during the same 2-week period and represent a change in functioning, and one of the symptoms must be either depressed mood or loss of interest/pleasure (Criterion A); the symptoms must not meet criteria for a mixed episode (Criterion B); the symptoms must cause clinically significant distress or impairment (Criterion C); the symptoms must not be due to the direct physiological effects of a substance or medical condition (Criterion D); and the symptoms must not be better accounted for by bereavement (Criterion E).

The CIDI also flagged instances in which respondents' symptoms met criteria for major depression but diagnostic criteria were not met because the symptoms were considered to be the result of direct physiological effects of using a substance (Criterion D). For conciseness, we have referred to this condition as "substance-induced depression". Criterion D was not met if participants endorsed that depressive symptoms were "always the result of taking medication, drugs or alcohol" or if they told a medical doctor about their depressive symptoms and the doctor said that the symptoms were caused by medication, drugs or alcohol. We were unable to assess DSM-IV Criterion $\mathrm{B}$, so participants with a mixed episode may have been included in the major depression group.
The severity of major depression was coded as mild (5-6 symptoms), moderate (7 symptoms) or severe ( $\geqslant 8$ symptoms).

\section{Help-seeking}

Participants who met DSM-IV criteria for major depression were asked whether they had received professional help for their depression in the previous year, and if so, whether they felt they had received as much help as they needed. They were asked about sources of help they had accessed and the main source of help received (general practitioner, drug treatment counsellor/worker, community health centre worker, private/ independent psychologist, private/independent psychiatrist, other private counsellor, or other).

\section{Substance use}

Methamphetamine dependence was diagnosed using the CIDI. ${ }^{8}$

The number of days of drug use in the previous month was measured using the $\mathrm{OTI}^{11}$ (daily substance use was defined as having used 20 or more days in the previous month).

Drug use in the previous year was also assessed for all major drug classes. For each drug, participants were asked whether they had used the drug in the previous 12 months, and if so, how often, with possible response categories being: no use, less than weekly, weekly, 2 days per week, 3-4 days per week, or $\geqslant 5$ days per week.

A polydrug-use score was calculated based on the number of other drug classes used in the previous year (drug classes included cannabis, heroin, cocaine, ecstasy, hallucinogens, inhalants, alcohol, tobacco and benzodiazepines).

\section{Other measures}

Physical and mental health were assessed using the SF-12. Physical or mental health disability was defined as having a score below 40 ( $>1$ SD below the normative mean) on the physical or mental health component scales of the SF-12, respectively. ${ }^{12}$

Participants' use of health services in the previous year was also assessed, including the number of visits to GPs.

\section{Statistical analyses}

Descriptive analyses were based on nonparametric tests (median comparison tests for continuous variables and Pearson's $\chi^{2}$ tests for categorical variables). An exploratory post-hoc analysis was undertaken to examine correlates of major depression and substance-induced depression. Logistic regression was used to adjust for covariates. All tests were two-sided. Significance was set at $P<0.05$. Data were analysed using STATA/SE software, version 11.1 (StataCorp, College Station, Tex, USA).

\section{RESULTS}

\section{Demographic and drug-use characteristics}

Demographic and drug-use characteristics of participants are summarised in Box 1 . Participants were typical of methamphetamine users entering treatment in Australia, ${ }^{13}$ with a predominance of single unemployed males, most of whom (83\%) had injected methamphetamine. The median age was 31 years (range, 16-54 years). Almost all participants (97\%) met DSM-IV criteria for methamphetamine dependence.

The reported frequency of methamphetamine use over the previous year was typically 3-4 days per week (39\%) or more often (38\%).

Participants had used a median of five other drug classes in the previous year (range, 1-9), the most common being tobacco (96\%), alcohol (87\%) and cannabis (87\%). Almost half the sample (47\%) reported smoking cannabis on a daily basis over the previous year, and 21\% reported drinking alcohol at this level. The use of other drugs in the previous year was also common (ecstasy [63\%], cocaine [48\%], heroin [35\%] and inhalants [19\%]), but less than $5 \%$ of the sample reported using them daily.

\section{Prevalence of depression}

Forty per cent (95\% CI, 35\%-44\%) of participants $(n=158)$ met DSM-IV criteria for a major depressive episode in the previous year. Symptoms were usually current (82\% of depressed participants had experienced symptoms in the previous month) and episodes were usually severe (79\% of the sample). The prevalence of major depression did not differ significantly by treatment modality.

A further 44\% (95\% CI, 39\%-49\%) of the sample $(n=176)$ met the symptom criteria for major depression (DSM-IV Criterion A), but were excluded from the diagnosis because their symptoms were always the result of substance use (DSM-IV Criterion D). This substance-induced depression had a similar symptom profile to major depression, but with lower levels of suicidal ideation and with fewer participants reporting 
1 Characteristics of participants with major depression or substance-induced depression

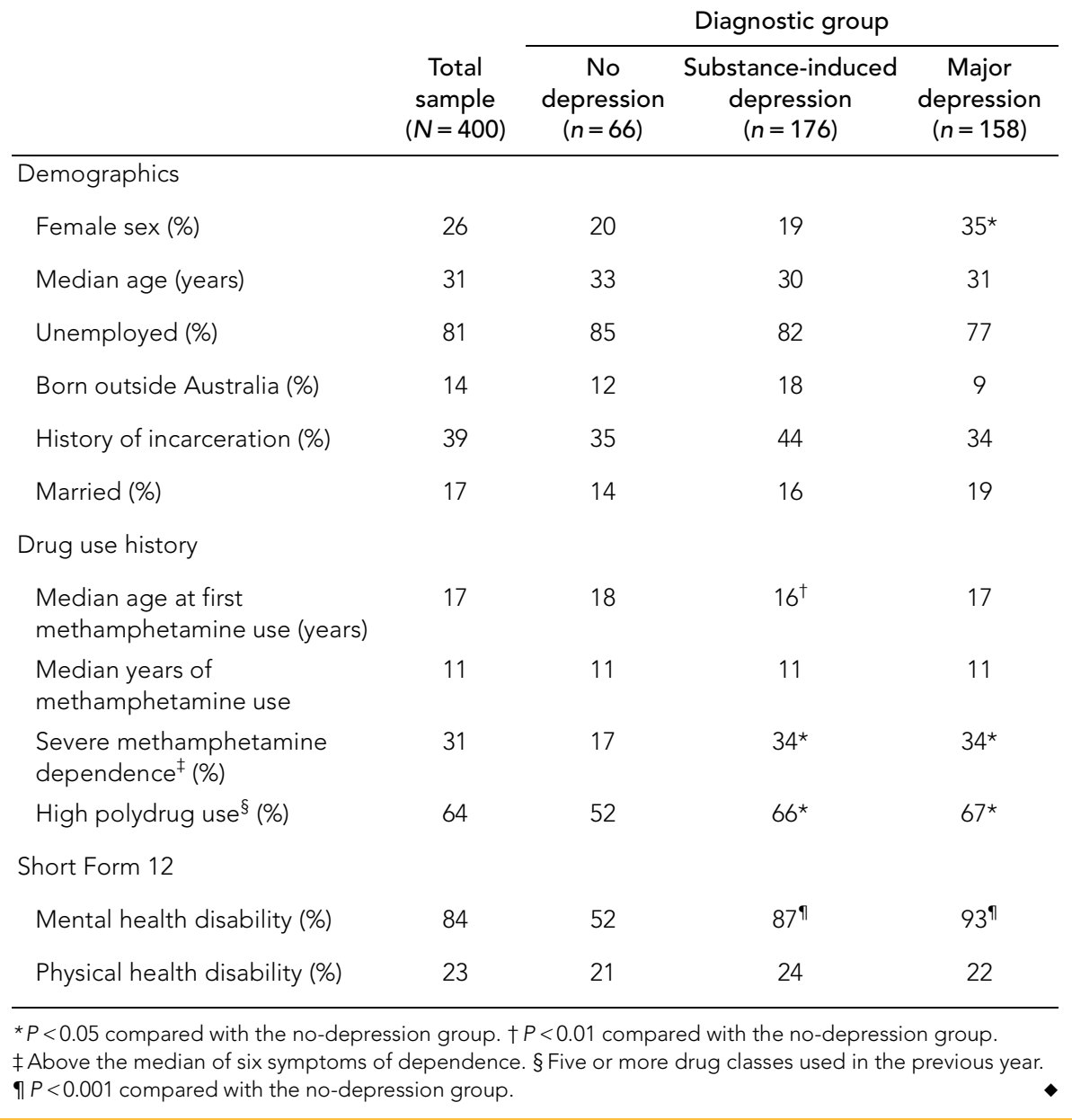

depressive symptom episodes that lasted 2 weeks or more (Box 2).

Both conditions were associated with high levels of disability due to poor mental health, more severe methamphetamine dependence and polydrug use (Box 2). Substance-induced depression was significantly associated with initiating methamphetamine use at a younger age, and major depression was more common among women (Box 1).

\section{Impact of polydrug use}

Specific polydrug patterns that were associated with depression were cannabis and benzodiazepine use. After adjustment for severe methamphetamine dependence and other demographic factors associated with depression (Box 1), both cannabis and benzodiazepine use remained significantly associated with major depression (cannabis: odds ratio [OR], 2.3 [95\% CI, 1.2-4.4]; P= 0.013; benzodiazepines: OR, 2.1 [95\% CI,
1.1-4.2]; $P=0.032$ ) and substance-induced depression (cannabis: OR, 2.4 [95\% CI, 1.24.6]; $P=0.010$; benzodiazepines: OR, 2.0 [95\% CI, 1.0-4.0]; $P=0.049$ ). The prevalence of major depression among participants who had used cannabis and/or benzodiazepines in the previous month was $42 \%$, compared with 26\% among those who had used neither drug.

\section{Disability}

Both major depression and substanceinduced depression were associated with high levels of mental health disability. Participants with major depression were nearly 13 times more likely to report mental health disability on the SF-12 than those without depression (OR, 12.5 [95\% CI, 5.6-28.1]; $P<0.001$ ), and those with substanceinduced depression had seven times the likelihood of mental health disability (OR, 7.0 [95\% CI, 3.5-13.8]; $P<0.001$ ). These effects were adjusted for other factors associ- ated with depression (ie, being female, earlier initiation into methamphetamine use, more severe methamphetamine dependence and higher polydrug use) that did not independently predict disability $(P>0.05)$.

\section{Help-seeking for major depression}

Fifty-eight per cent of participants with major depression $(n=91)$ had received professional help for their depression in the previous year. Of these, only $27 \%$ felt they had received as much help as they needed. Most participants (76\%) who did not receive any help felt that they needed help.

Participants who received help for depression cited their main source of help as GPs (42\%), drug treatment professionals (16\%), private psychiatrists (11\%), psychologists (9\%) and counsellors (8\%). Ninety per cent of participants with major depression had seen a GP in the previous year, with a median number of five visits.

\section{Antidepressant use}

About half (53\%) of participants who had experienced a major depressive episode in the previous year, and $27 \%$ of those who had not, had taken antidepressants. Polydrug use among participants who were taking antidepressant medication was ubiquitous. Of those who had used antidepressant medication in the previous month (21\%), most (69\%) had taken antidepressants every day during this time, almost all (93\%) had continued to use methamphetamine, and the majority had done so frequently (median, 16 days of use in the previous month). Most also used cannabis (79\%) and did so on a regular basis (median, 18 days of use in the previous month). Alcohol use was also common (74\%), with $21 \%$ of users drinking daily while taking antidepressants.

\section{DISCUSSION}

Our findings suggest that clinical levels of depression are almost ubiquitous among people entering treatment for methamphetamine use, indicating a need for clinical protocols to manage depression in this context. Four in 10 methamphetamine treatment entrants in the current sample met DSM-IV criteria for a major depressive episode in the previous year, and a further 44\% had substance-induced depressive symptoms that were similarly severe and disabling. These levels of depression are high, even when compared with levels in other drug-dependent populations. ${ }^{3}$ 


\begin{tabular}{lcc}
$2 \begin{array}{l}\text { Comparison of symptoms for major depression and substance-induced } \\
\text { depression }\end{array}$ & $\begin{array}{c}\text { Substance-induced } \\
\text { depression }(n=176)\end{array}$ & $\begin{array}{c}\text { Major depression } \\
(n=158)\end{array}$ \\
\hline Periods of feeling sad, empty or depressed & $79 \%$ & $94 \%^{\star}$ \\
Loss of interest & $97 \%$ & $96 \%$ \\
Appetite change & $96 \%$ & $95 \%$ \\
Sleep disturbances & $98 \%$ & $97 \%$ \\
Slowness or restlessness & $78 \%$ & $79 \%$ \\
Fatigue or loss of energy & $89 \%$ & $94 \%$ \\
Feeling worthless or guilty & $90 \%$ & $80 \%{ }^{\dagger}$ \\
Trouble concentrating & $100 \%$ & $100 \%$ \\
Suicidal ideation & $61 \%$ & $73 \%{ }^{\dagger}$ \\
\hline$* P<0.001, \dagger P<0.01$ for difference between groups. & &
\end{tabular}

Distinguishing between major depression and substance-induced symptoms of depression was problematic. Whether existing structured psychiatric assessment instruments can accurately make this distinction remains controversial. ${ }^{14-16}$ The differential diagnosis of independent and substance-induced disorders relies on respondents having insight into the temporal relationship between their substance use and their depressive symptoms, which, in turn, requires periods of abstinence from drug use. Long periods of abstinence are a rarity in populations of heavily dependent drug users, who, by definition, find it hard to abstain, and typically engage in almost daily patterns of polydrug use, oscillating between intoxication and withdrawal. Making a diagnosis of depression in the context of methamphetamine use is particularly problematic, as many of the drug's acute effects (such as reduced appetite and insomnia) and withdrawal symptoms (such as anhedonia, depressed mood, hypersomnolence and increased appetite) overlap with the symptoms of depression. ${ }^{17}$ Participants in our study may have misattributed depressive symptoms to drug use, or vice versa.

GPs were the most common source of help for depression among methamphetamine users, but even so, a large proportion of methamphetamine users did not receive help for depression from their GP (or via other avenues), or did not receive as much help as they thought they needed. This finding indicates that there is a substantial gap in mental health service provision for dependent methamphetamine users and that GPs are well placed to provide treatment and referral for this population. The use of antidepressant medication was common in this sample of methamphetamine users, and GPs need to consider the potential interactions between antidepressant medication and the range of illicit drugs that are concurrently consumed by methamphetamine users. Particular consideration should be given to the role of psychosocial interventions for depression (eg, cognitive behaviour therapy) as an alternative to medication. ${ }^{2,7}$

There is undoubtedly a range of factors that contribute to depression among methamphetamine users. Illicit drug use is associated with various social and individual risk factors for depression (eg, social stressors, poverty, and physical illness such as hepatitis C). In our study, concurrent polydrug use (in particular, use of cannabis and benzodiazepines) was significantly associated with depression, a finding that is consistent with previous research in this area. ${ }^{1}$ The relationship between drug use and depression is likely to be bidirectional, with heavy drug dependence leading to mood changes and life stressors that increase the risk of depression, and people with depression self-medicating with drugs to relieve their depression. This is particularly likely in the context of methamphetamine use, because acute intoxication with the drug has a potent shortterm antidepressant effect. ${ }^{18}$

In conclusion, the clinical management of depression and related suicide risk is imper- ative within settings that provide treatment for methamphetamine use, because of the high rates of depression seen in this context. Further research is needed to understand whether methamphetamine use increases the risk for major depression or whether the high rates of depression in this population are a consequence of transient drug-related depressive symptoms. Finally, caution is needed when diagnosing major depression in the context of methamphetamine dependence.

\section{ACKNOWLEDGEMENTS}

The data reported here were collected through the Methamphetamine Treatment Evaluation Study (MATES). The study was conducted by the National Drug and Alcohol Research Centre, University of New South Wales, and was funded by the National Health and Medical Research Council and the Australian Government Department of Health and Ageing. We acknowledge the contribution of the following MATES investigators: Robert Ali, Amanda Baker, Sharon Dawe, Richard Mattick, Abdullah Mamun and Jake Najman. We also thank the research officers who assisted with data collection (Shelley Cogger, Erin Kelly, Kate Hetherington, Grace Ho, Julia Rosenfeld, Cathie Sammut, Sagari Sarkar, Rachel Sutherland and Miriam Wyzenbeek), the participating treatment agencies, and the research participants.

\section{COMPETING INTERESTS}

Rebecca McKetin has received consultancy fees from the United Nations Office on Drugs and Crime. Daniel Lubman has received speakers fees from Bristol-Myers Squibb (2007) and conference travel support from AstraZeneca and Janssen (2008). Nicole Lee has done paid consultancy work for St John of God Health Care.

\section{AUTHOR DETAILS}

Rebecca McKetin, BSc(Psych)(Hons), PhD, Fellow in Mental Health Research ${ }^{1,2}$

Daniel I Lubman, FRANZCP, FAChAM, PhD, Director, ${ }^{3}$ and Professor of Addiction Studies and Services ${ }^{4}$

Nicole M Lee, BSc(Hons), MAPS, PhD, Associate Professor ${ }^{5}$

Joanne E Ross, BSc(Hons), PhD, Senior Lecturer $^{2}$

Tim N Slade, BSc(Psych), PhD, Senior Research Fellow ${ }^{2}$

1 Centre for Mental Health Research, Australian National University, Canberra, ACT.

2 National Drug and Alcohol Research Centre, University of New South Wales, Sydney, NSW.

3 Turning Point Alcohol and Drug Centre, Melbourne, VIC.

4 Monash University, Melbourne, VIC.

5 National Centre for Education and Training on

Addiction, Flinders University, Adelaide, SA.

Correspondence:

rebecca.mcketin@anu.edu.au 


\section{REFERENCES}

1 Riehman KS, Iguchi MY, Anglin MD. Depressive symptoms among amphetamine and cocaine users before and after substance abuse treatment. Psychol Addict Behav 2002; 16: 333-337.

2 Rawson RA, Gonzales R, Brethen R. Treatment of methamphetamine use disorders: an update. J Subst Abuse Treat 2002; 23: 145-150.

3 Hasin D, Nunes EV, Meydan J. Comorbidity of alcohol, drug, and psychiatric disorders: epidemiology. In: Kranzler HR, Tinsley JA, editors. Dual diagnosis and psychiatric treatment: substance abuse and comorbid disorders. 2nd ed. New York: Marcel Dekker, 2004: 1-34.

4 Bamford NS, Zhang $H$, Joyce JA, et al. Repeated exposure to methamphetamine causes long-lasting presynaptic corticostriatal depression that is renormalized with drug readministration. Neuron 2008; 58: 89-103.

5 London ED, Simon SL, Berman SM, et al. Mood disturbances and regional cerebral metabolic abnormalities in recently abstinent methamphetamine abusers. Arch Gen Psychiatry 2004; 61: 73-84.

6 Glasner-Edwards S, Marinelli-Casey P, Hillhouse $M$, et al. Depression among methamphetamine users: association with outcomes from the
Methamphetamine Treatment Project at 3-year follow-up. J Nerv Ment Dis 2009; 197: 225-231.

7 Baker A, Lee NK, Claire M, et al. Brief cognitive behavioural interventions for regular amphetamine users: a step in the right direction. Addiction 2005; 100: 367-378.

8 Janca A, Robins LN, Cottler LB, et al. Clinical observation of assessment using the Composite International Diagnostic Interview (CIDI). An analysis of the CIDI Field Trials - Wave II at the St Louis site. Br J Psychiatry 1992; 160: 815-818.

9 American Psychiatric Association. Diagnostic and statistical manual of mental disorders. 4th ed. Text revision. Washington, DC: APA, 2000.

10 Haro JM, Arbabzadeh-Bouchez S, Brugha TS, et al. Concordance of the Composite International Diagnostic Interview version 3.0 (CIDI 3.0) with standardized clinical assessments in the WHO world mental health surveys. Int J Methods Psychiatr Res 2006; 15: 167-180.

11 Darke S, Hall W, Wodak A, et al. Development and validation of a multi-dimensional instrument for assessing outcome of treatment among opiate users: the Opiate Treatment Index. Br J Addict 1992; 87: 733-742.

12 Ware JE, Kosinksi MM, Keller SD. How to score the SF-12 physical and mental health summary scales. Boston: The Health Institute, New England Medical Centre, 1995.

13 McKetin R, Kelly E, Indig D. Characteristics of treatment provided for amphetamine use in New South Wales, Australia. Drug Alcohol Rev 2005; 24: 433-436.

14 Hasin D, Samet S, Nunes E, et al. Diagnosis of comorbid psychiatric disorders in substance users assessed with the Psychiatric Research Interview for Substance and Mental Disorders for DSM-IV. Am J Psychiatry 2006; 163: 689-696.

15 Kranzler HR, Kadden RM, Babor TF, et al. Validity of the SCID in substance abuse patients. Addiction 1996; 91: 859-868.

16 Bryant KJ, Rounsaville B, Spitzer RL, et al. Reliability of dual diagnosis. Substance dependence and psychiatric disorders. J Nerv Ment Dis 1992; 180: 251-257.

17 McGregor C, Srisurapanont M, Jittiwutikarn J, et al. The nature, time course and severity of methamphetamine withdrawal. Addiction 2005; 100: 1320-1329.

18 De Rudolf GM. The treatment of depression with methylamphetamine. J Ment Sci 1956; 102: 358-363.

(Received 3 Sep 2010, accepted 18 Apr 2011) 